\title{
Study of Electrical, Thermal and Optical Behavior of Polypyrrole Filled PVC:PMMA Thin Film Thermoelectrets
}

\author{
VIJAYA S. SANGAWAR and NEERAJA A. MOHARIL
}

Polymer Research Laboratory, Department of Physics, Govt. Institute of Science and Humanities, Amravati-444604, India

haridasneeraja@rediffmail.com

Received 21 May 2012 / Accepted 13 June 2012

\begin{abstract}
The present study reveals with study of electrical, thermal and optical band gap of polypyrrole filled PVC: PMMA thin films themoelctrets. Conducting polymer composites have attracted considerable interest in recent years because of their numeric applications in variety of electric and electronic devices. Polypyrrole has been regarded as one of the most studied conducting polymer because of its physical and electrical properties that have led to several applications such as solid state devices and electronics. The electrical conductivity of polypyrrole filled PVC: PMMA thermoelectrets have been studied. Electret effects in polymers can be produced by orientation of dipoles and /or trapping of charge carriers injected from electrodes as well as generated in the bulk of polymers. It has been shown by thermally stimulated discharge conductivity technique (TSDC) that the characteristics of electrets are very sensitive to the structure of electret forming materials. In this respect TSDC study of composite is likely to yield information about the extent of mixing between the components. Electrets prepared by composites have better charge storage capacity than the individual polymers. Measurements of TSD conductivity have been carried out at different polarizing fields. It has been observed that the conductivity of polypyrrole filled PVC: PMMA composite have been increased with increase in percentage of polypyrrole. The X-RD diffractogram reveals the amorphous nature of the films. The thermograms are plotted between $\log \sigma$ and temperature $\left(10^{3} / \mathrm{T}\right)$.From the analysis of the absorption spectra the band gap of polypyrrole filled PVC: PMMA composite have been found to be lie in the range $1.8 \mathrm{eV}$ to $3.3 \mathrm{eV}$. Thermal stability of polypyrrole filled PVC: PMMA was investigated by TGA/DSC. It is evident from the results that PPy filled polyblends are more stable.
\end{abstract}

Keywords: TSDC, Thermoelectrets, Optical band gap, Polypyrrole, TGA/DSC

\section{Introduction}

Polypyrrole is an intrinsic conducting polymer which can be made to have conductivities up to $1000 \mathrm{~S}^{-\mathrm{cm}^{-1}}$ rendering its versatile applications in batteries, electronic devices, functional electrodes, electro chromic devices, optical switching devices, sensors and so on. In recent years, polypyrrole is popular in research and has been focus of many studies over other conducting polymers because of its high chemical and air stability and ease of preparation ${ }^{1}$. The typical polypyrrole, which is insoluble and infusible, exhibits poor processability and 
lacks essential mechanical properties. Efforts to overcome these drawbacks have led to numerous researchers on the synthesis of polypyrrole. Among them, a significant strategy to approach high electrical conductivity is preparing blends or composites of polypyrrole polymers with other insulating polymers. The combination of conventional polymers with conductive polymers or fillers is an important alternative to obtain new polymeric materials with design properties. In such blends, the insulating polymer provides good mechanical properties and processability while the conducting polymer would provide electrical conductivity. In addition, through blending, the brittleness and lack of processability that are the main drawbacks hindering conducting polymers better utilization could be overcame by new polymeric materials with improved processability, flexibility and controllable conductivity ${ }^{2}$.

The present study discusses the electrical, thermal and optical properties in PPy filled PVC:PMMA thin film thermoelectrets. Since PVC is proton donor and PMMA is proton acceptor selected as a basic polymeric materials. The choice of these polymers is taken into account for the donor acceptor capabilities. The conducting polymer, polypyrrole is added into these polymers to identify electrical conduction. In number of applications band study is essential, for light emitting diode, transparency in the visible region combined with high electrical conductivity ${ }^{3,4}$. Sangawar et al have studied the band gap determination of PVCCharcoal composite thin film ${ }^{5}$. Shaktawat et al have studied the electrical conductivity and band gap determination of polypyrrole doped with different acids ${ }^{6}$.

\section{Experimental}

Polypyrrole was synthesized by chemical oxidative method from pyrrole monomer using ammonium per sulfate as oxidant and $p$-Toluene sulphonic acid as a dopant. Aqueous solution of pyrrole $0.5 \mathrm{~mL}$ in $7.5 \mathrm{~mL}$ water was added instantly to a solution of ammonium per sulphate $(150 \mathrm{mg})$ and $p$-toluene sulphonic acid $(500 \mathrm{mg})$. After 5 minutes the product was recovered by filtration, washed with water and dried at $70{ }^{\circ} \mathrm{C}$ for 12 hours ${ }^{7}$.

\section{Preparation of sample}

Polymethyl methacrylate was supplied by dental products India Ltd. (Mumbai). Polyvinyl chloride supplied by Reliance Industries (Mumbai). Pyrrole monomer (Emerck Germany) was supplied by G. Kuntal \& Company (Mumbai).

In the present study PPy acts as an additive. To prepare the samples containing the two polymers and additives the following procedure is adapted. For the composite sample of 1 weight percent $0.78 \mathrm{~g}$ of PVC and $0.19 \mathrm{~g}$ of PMMA was dissolved in $30 \mathrm{~mL}$ of THF and $0.01 \mathrm{~g}$ of PPy was then added in the solution which was kept at $343 \mathrm{~K}$ for $12 \mathrm{~h}$ with continuous stirring for uniform dispersion. After stirring the solution for specific hours the film were prepared by pouring the solution on a clean optically plane glass plate. This glass plate was kept over a pool of mercury to ensure uniform thickness. The whole assembly was placed in a dust free chamber. After complete evaporation of solvent the film was detached from glass surface ${ }^{8}$.

\section{Thickness measurement}

The thickness of the sample was measured by the compound microscope in conjunction with an occulometer having a least count of $15.38 \mu \mathrm{m}$ similar to method reported by Sangawar ${ }^{5}$. The thickness of the sample was kept constant throughout the work and is of the order of $46.14 \mu \mathrm{m}$.

\section{Electrode coating}

The electrode coating on the film of measured thickness was done by using quick drying and highly conducting silver paint supplied by Eltecks corporation, Bangalore. A mask of 
circular aperture of $2.5 \mathrm{~cm}$ diameter was used while coating, to ensure uniformity in the size of the coated silver electrode.

\section{Structural characterization}

For structural characterization the x-ray diffractograms of PPy filled PMMA: PVC films were obtained on XRD-PKL 1729 Phillips Bulk XRD (Figure 1). The x-ray diffractograms of all the samples ensures the amorphous nature. The presence of PPy in PMMA was confirmed by SEM of the sample taken on Jeol JSM 6380A (Figure 2).

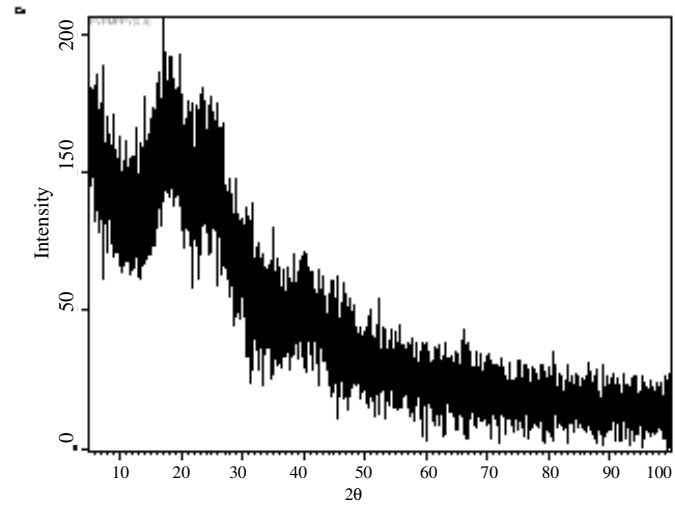

Figure 1. X-ray diffraction pattern for $0.5 \mathrm{wt} \%$ PPy filled PVC: PMMA thin films

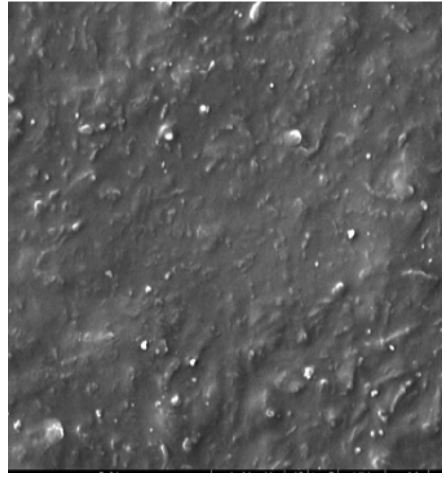

Figure 2. SEM micrograph of PPy filled PMMA:PVC thin films

\section{Measurement of thermally stimulated discharge conductivity (TSDC)}

Thermo electrets preparation: Experimental sample was sandwiched between two brass electrodes of the sample holder. The metal polymer metal (M-P-M) system so formed was placed inside the furnace. The M-P-M system was heated at a nearly uniform rate up to a polarizing temperature $\mathrm{Tp}=343 \mathrm{~K}$ and was kept constant for 30 minutes. Different polarizing fields $(\mathrm{Ep}), 4 \mathrm{kV} / \mathrm{cm}, 8 \mathrm{kV} / \mathrm{cm}, 12 \mathrm{kV} / \mathrm{cm}$ and $16 \mathrm{kV} / \mathrm{cm}$ were applied using a stabilized D.C. voltage source for $1 \mathrm{~h}$ keeping the field on. The sample was slowly cooled to room temperature under continuing electric stresses. Total time of polarization was adjusted to be $2.5 \mathrm{~h}$ in each case. After polarization, the field was removed and the sample was short circuited for 20 minutes in order to remove stray charges if any. Thus thermoelectres were prepared by simultaneous application of heat and different polarizing fields ${ }^{9}$.

After the electret formation the M-P-M assembly was placed in a controlled temperature furnace supplied by Pushpa Scientific, Hyderabad. The sample was thermally discharged at a uniform rate of $2 \mathrm{~K} / \mathrm{min}$ and the corresponding was measured in the temperature region 313-403. The transmission spectra of polypyrrole filled PVC: PMMA recorded in the region of wavelength 200-700 $\mathrm{nm}$ on Hitachi-330UV-VIS spectrophotometer at room temperature.

\section{Results and Discussion}

Figure 1 shows x-ray diffraction pattern ( $2 \theta$ versus intensity) polypyrrole filled PVC: PMMA thin films. The diffraction pattern shows big humps at low diffraction angles, which confirms the amorphous nature of polymer composites ${ }^{10}$. 
Figure 2 shows SEM micrographs of PPy filled polypyrrole. The scanning electron microscopy shows the non uniform distribution of polypyrrole in PMMA: PVC polyblend. The PPy particles are distributed in the polyblend which confirms the presence of polypyrrole in the polyblend.

Figure 3 and 4 shows the thermograms for the thermoelectrets of unfilled and PPy filled PMMA:PVC for different polarizing fields $(4,8,12$ and $16 \mathrm{kV} / \mathrm{cm})$ in the temperature range 313-403 K. The conductivity increases with increasing temperature by an equation

$$
\sigma=\sigma_{0} \exp \left(-\mathrm{E}_{\mathrm{a}} / \mathrm{KT}\right)
$$

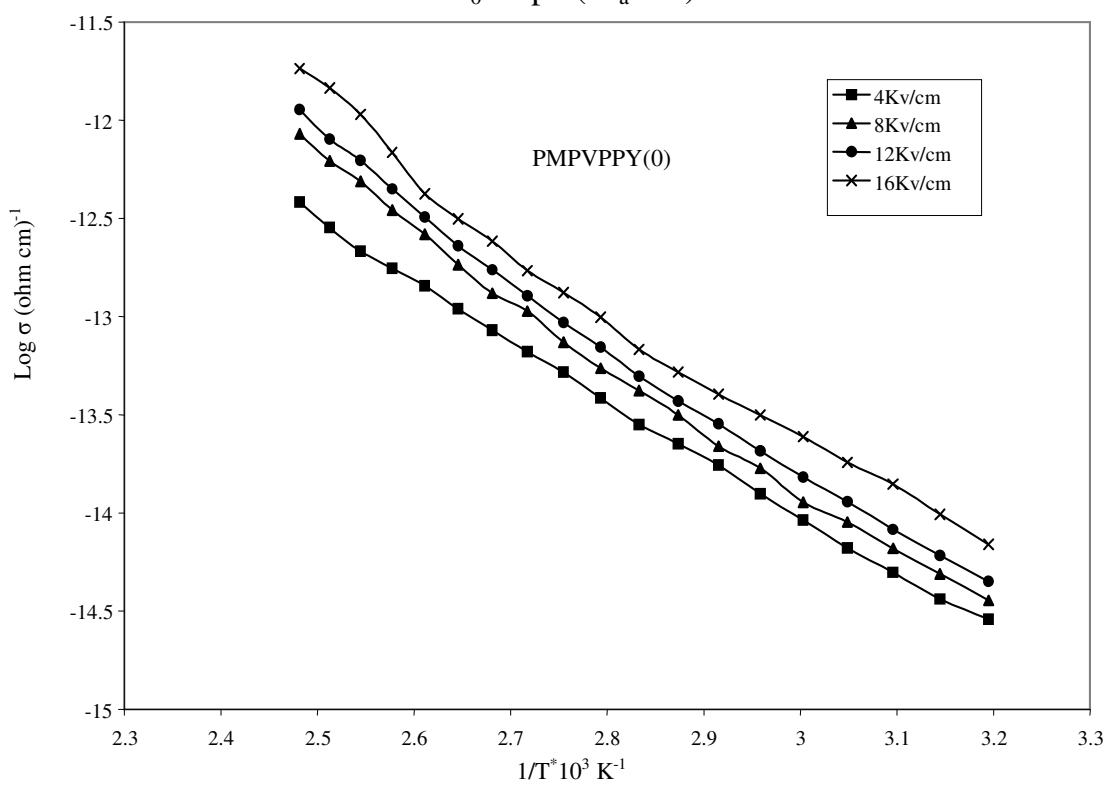

Figure 3. Thermograms of unfilled PVC: PMMA

Where $\sigma_{0}$ is pre exponential factor, $E_{a}$ activation energy of conduction and $K$ is the Boltzman's constant. PVC and PMMA are amorphous polymers but have different electrical conductivities. The aim of the study is to observe the effect on conductivity of PVC: PMMA blend when PPy added in different weight percent and along with the effect of polarizing field (4, $8,12$ and $16 \mathrm{kV} / \mathrm{cm})$.

Electrical conductivity of PPy involves movement of positively charged carriers or electrons along polymer chain and hopping of these carriers between polymeric chains. Polyvinyl chloride is proton donor and polymethyl methacrylate is proton acceptor. The charge transfer occurs due to increase in electro positivity. The degeneracy of the ground state has an important effect on the nature of charge species. Polypyrrole is nondegenerate ground state polymer. On addition of polypyrrole the polymer chain gets ionized and this ionization process creates polarons having half spin. At low doping level these polarons are carriers of electricity. Increased polarons result in large probability of interaction which in turn results to form bipolaron. Bipolarons are doubly charged but spinless. A single bipolaron is more stable than double polarons. The relatively high conductivity of polymers probably results from motion of spinless bipolarons. It is also evident that the conductivity also increases with the polarizing field ${ }^{11,12}$. 
The activation energy values are calculated from the slope of thermograms. It is observed that the value of activation energy decreases with increase in concentration of PPy in PVC: PMMA and is found to be $0.61 \mathrm{eV}$. This is in good agreement with the reported order of magnitudes ${ }^{13}$.

It can be noted that the room temperature electrical conductivity depends strongly on the fraction of polypyrrole in the blends. The conductivities of these blends increase with increasing content of conductive polypyrrole showing the conductive fractions dependence of the conductivity. The enhancement of conductivity of the blends films with increasing polypyrrole loading is owing to the conductive paths formation through the blends ${ }^{14}$. This shows percolation of polypyrrole in the polyblends result in increase in the conductivity.

The optical transmission spectra of (1) PVC: PMMA, (2) PVPMPPY 0.5 and (3) PVPMPPY 7 as shown in Figure 4. The absorption coefficient was calculated as a function of photon energy from transmission $v s$. wavelength curve ${ }^{16}$. The absorption $\mathrm{A}$ is defined as

$$
\mathrm{A}=\alpha \mathrm{h} v
$$

Where $\alpha$ is the absorption coefficient, $\mathrm{h}$ is the Planck's constant and $v$ is the frequency. The absorption coefficient $\alpha$ is directly determined by the relation

$$
A=(2.303 / d) \log (1 / T)
$$

Where $\mathrm{d}$ is the film thickness and $\mathrm{T}$ is the transmittance. The variation in the absorption coefficient with photon energy, hv is given by

$$
\alpha h v=\beta[h v / \operatorname{Eg}(\mathrm{opt})]^{\mathrm{m}} / \mathrm{hv}
$$

Where Eg (opt) is the optical band gap energy, $\beta$ is the temperature dependent and describes the degree of randomness of the material ${ }^{15}$. Figure 5 shows the plot of $(\alpha h v)^{1 / 2} v s$. hv. The extrapolation of the straight line to $(\alpha \mathrm{hv})^{1 / 2}=0$ axis gives the value of band gap energy ${ }^{16}$. From the analysis of these graphs the value of band gap energy for unfilled PVC: PMMA, 0.5wt\% polypyrrole filled PVC: PMMA and $7 \mathrm{wt} \%$ polypyrrole filled PVC: PMMA comes out as $3.21 \mathrm{eV}, 2.5 \mathrm{eV}$ and $1.8 \mathrm{eV}$ respectively.

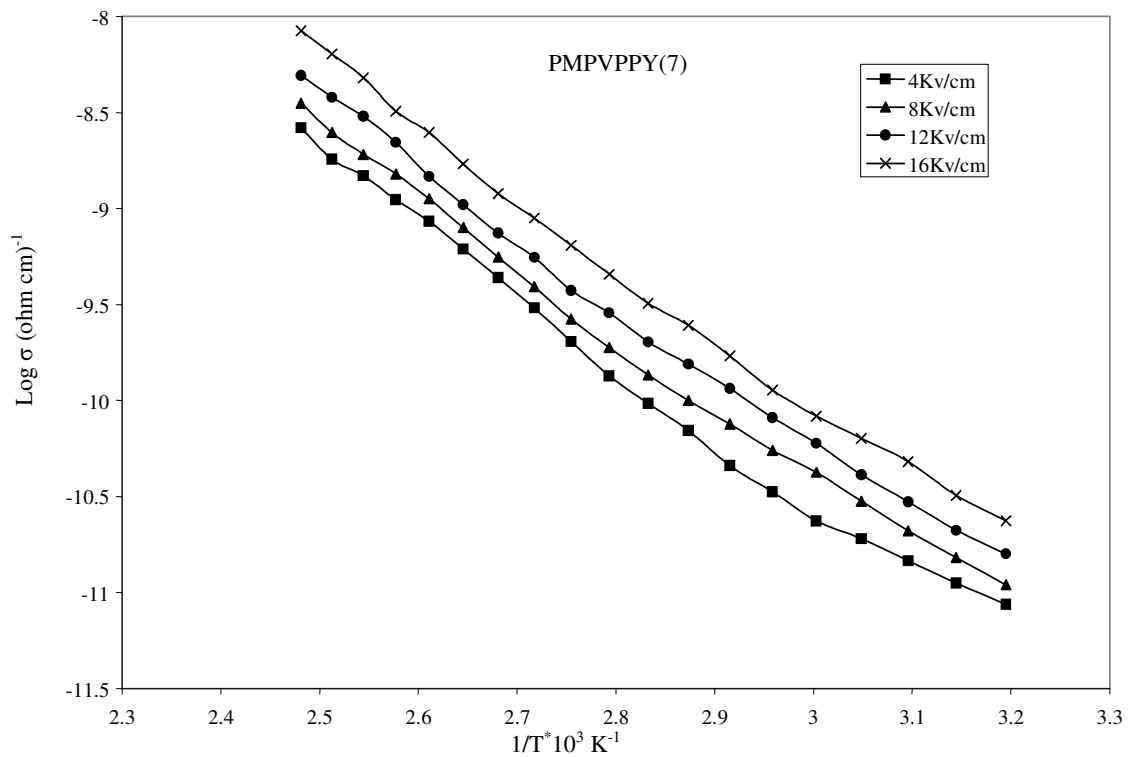

Figure 4. Thermograms of PPy filled PVC: PMMA 


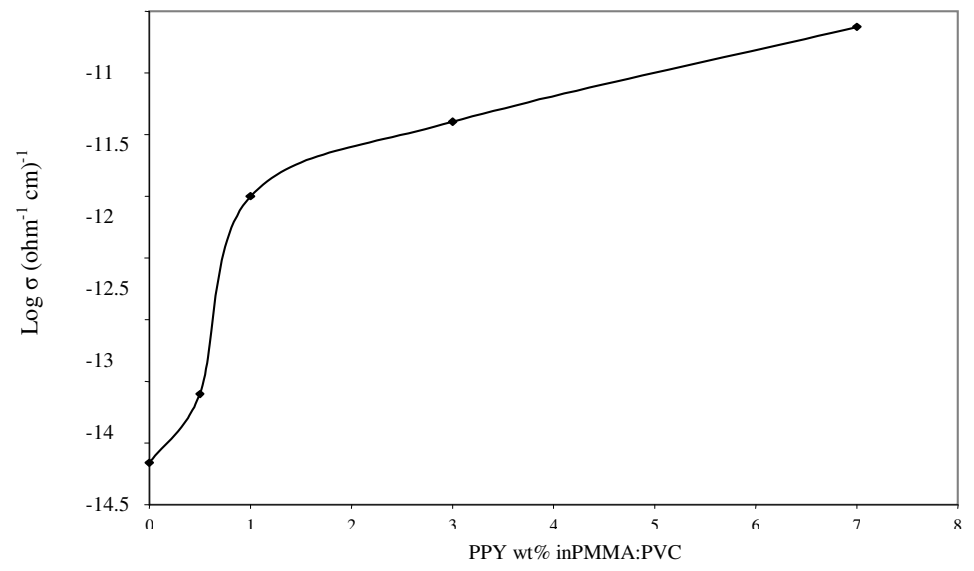

Figure 5. Shows Plot of electrical conductivity versus wt\% of PPY in PMMA: PVC thin films at room temperature

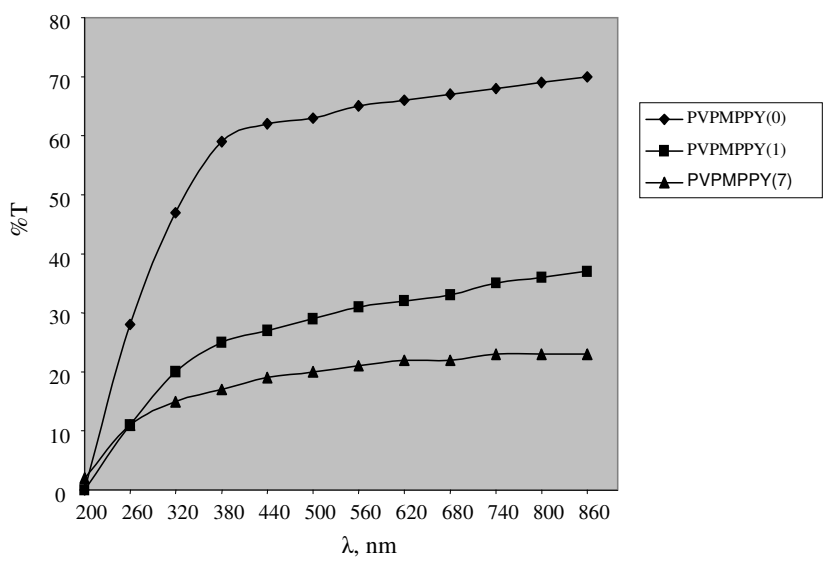

Figure 6. Optical transmission spectra of unfilled PVC: PMMA and PPY filled PVC: PMMA

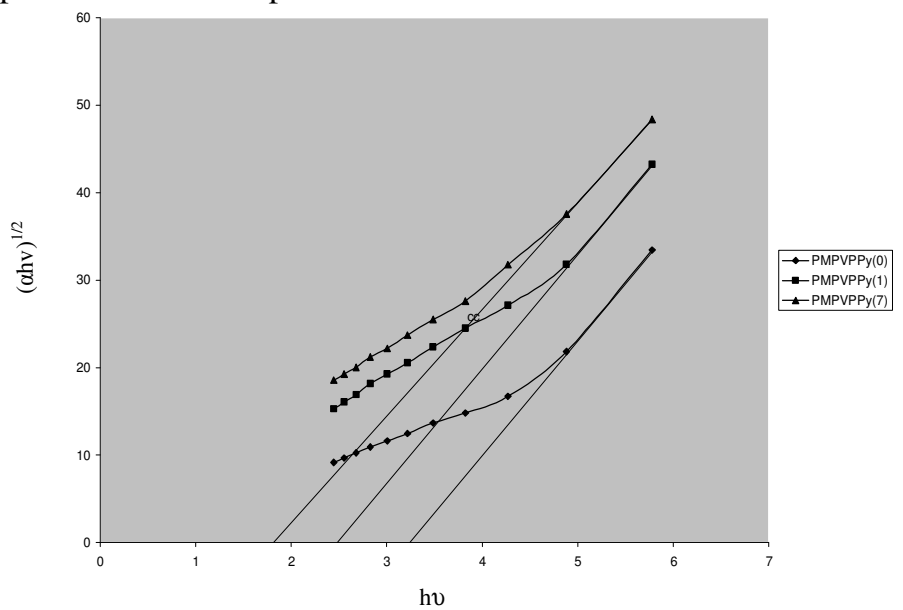

Figure 7. Plots of $(\alpha h v)^{1 / 2} v s$. hv for unfilled PVC: PMMA and PPY filled PVC: PMMA 
The lower values of Eg for higher weight percent addition of polypyrrole may be attributed to the creation of localized states in the band gap as a result of compositional disorder. Also the increase in the number of unsaturated defects increase the density of localized states in the band structure and consequently leads to decreasing the optical gap ${ }^{17}$.

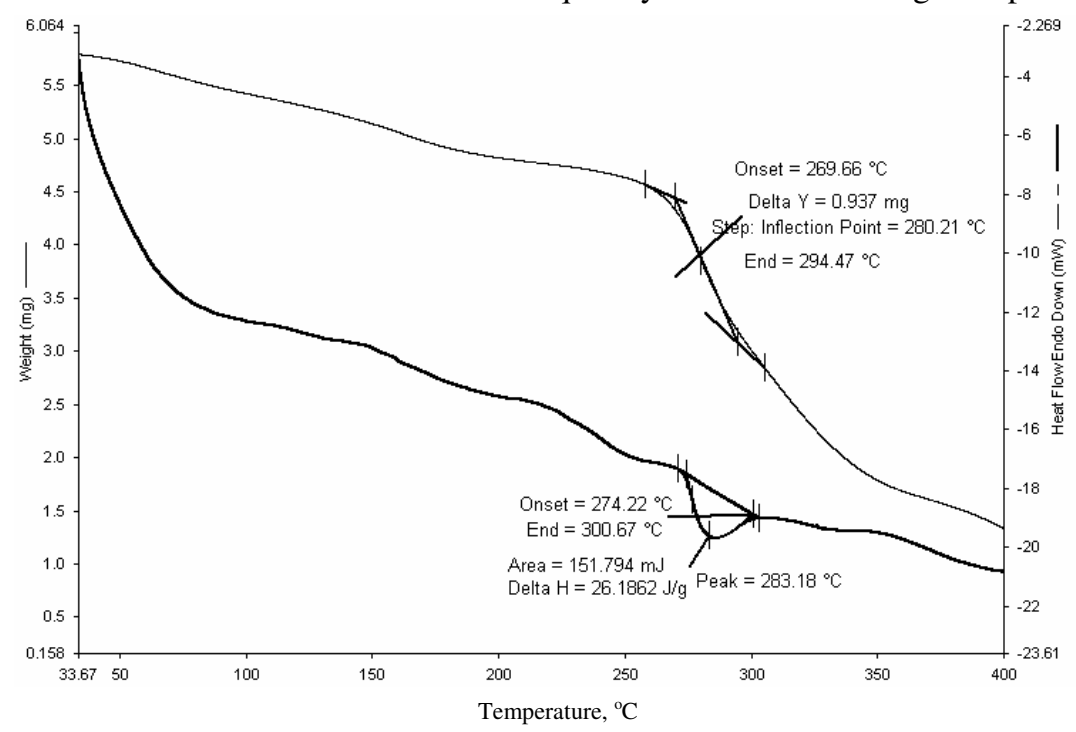

Figure 8. TGA/DSC curves for unfilled PVC: PMMA thin films

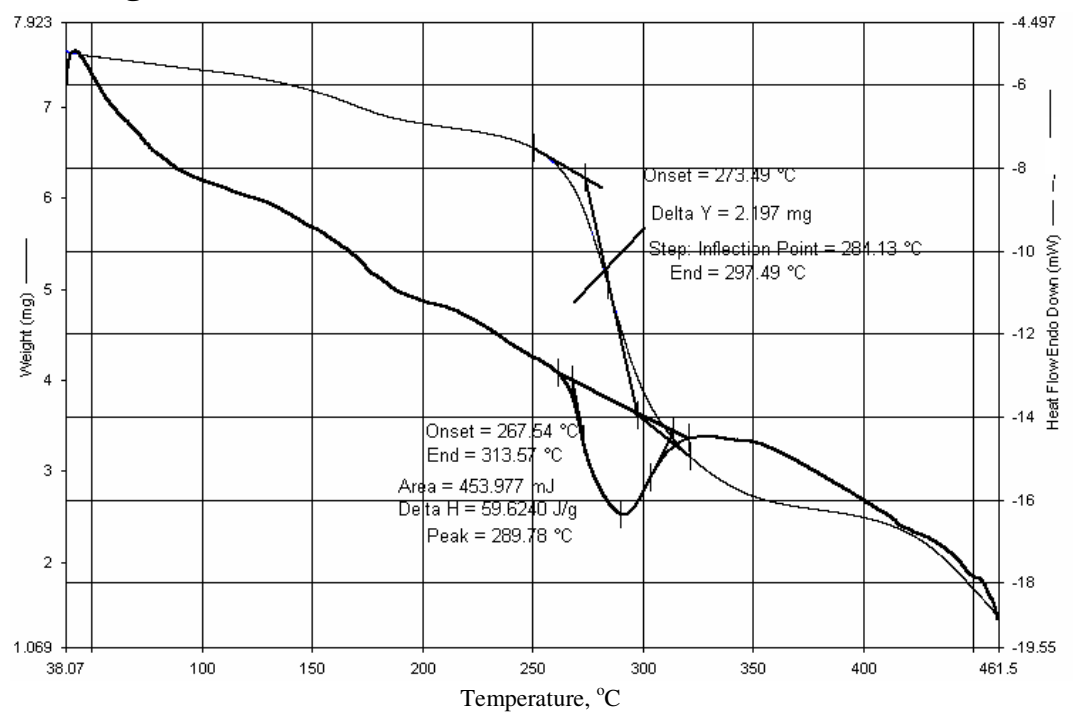

Figure 9. TGA/DSC curves for PPY filled PVC: PMMA Thin films

From the TGA curves it is observed that the onset temperature increases from $269^{\circ} \mathrm{C}$ to $273{ }^{0} \mathrm{C}$. The mass loss for $0.5 \mathrm{wt} \%$ PPy filled PVC: PMMA polyblend starts at $269.66{ }^{0} \mathrm{C}$ and that for $7 \mathrm{wt} \%$ starts at $273.49{ }^{\circ} \mathrm{C}$. It is observed that the mass continues to vary slowly at temperature below $400{ }^{\circ} \mathrm{C}$. Above this temperature, this process takes place very rapidly. The mass loss at onset temperature decreases from $16 \%$ to $15 \%$. 
From the DSC curves, three major transitions corresponding to glass transition temperature, melting temperature and vaporization temperature were observed for different concentration of PPy. In this study we have not focused on vaporization temperature since almost all polymers will thermally degrade before they vaporize. The endothermic peak and the broad peaks on DSC curves correspond to melting temperature and glass transition temperature $^{18}$. The corresponding melting temperatures for $0.5 \mathrm{wt} \%$ and $7 \mathrm{wt} \% \mathrm{PPy}$ concentrations are $283.18{ }^{\circ} \mathrm{C}$ and $289.78{ }^{\circ} \mathrm{C}$. The glass transition temperature increases from 99 to $100{ }^{\circ} \mathrm{C}$. From the data it is apparent that the thermal degradation began to occur only after the materials have absorbed certain amount of heat energy ( $\Delta \mathrm{H}$ values). The heat initiated the degradation process and breaking down of molecular chain ruptures ${ }^{19}$.

From the TGA data it is observed that the weight loss of polypyrrole filled composites decreases and the thermal degradation temperature increases with increase in concentration of polypyrrole in the polymers.

The TGA/DSC analysis for polypyrrole filled composites shows that the glass transition temperature slightly decreases with increase in concentration of filler. This shows that polypyrrole filled PVC:PMMA polyblends are more stable.

\section{Conclusion}

Present study reveals that the temperature, polarizing field and concentration of PPy influences the conductivity of PVC: PMMA blends. The order of D.C. electrical conductivity of unfilled PVC: PMMA was of the order of $10^{-13} \mathrm{ohm}^{-1} \mathrm{~cm}^{-1}$. After doping of PPy in PVC:PMMA in sufficient quantity it is increases to $10^{-7} \mathrm{ohm}^{-1} \mathrm{~cm}^{-1}$. The increase in conductivity at room temperature is due to the percolation of PPy in the polyblends. The optical band gap energy decreases with increase in percentage of polypyrrole in PVC: PMMA. The optical absorption shows that the polypyrrole filled films have allowed direct transitions. The TGA/DSC analysis shows that the films are thermally more stable. It can be concluded that the wt.\% of conductive fractions, temperature, charge mobility and the conductivity behavior of the films are in tight interaction with each other. The addition of conductive fillers in the insulating polymers could lead to innovation in synthetic method for better conducting films.

\section{Acknowledgement}

The authors are thankful to Pune University, Pune for providing UV-VIS facilities.

\section{References}

1. Kassim A, Sagadavan M, Adzmi F and Mahmud Ekramul H N H, Mater Sci., 2004, 10, 255.

2. Das B, Kumar S, Chakraborty S, Chakraborty D and Gangopadhyay S, J Appl Polym Sci., 1998, 69, 841-844.

3. Greeham N C, Moratti S C, Bradley D D C, Friend R H and Holmes A B, Nature 1993, 365, 628.

4. Kobayashi M, Colaneri N, Boysel M, Wudl F and Heeger A J, J Chem Phys., 1985, 82, 5717-5723.

5. Sangawar V S, Chikhalikar P S, Dhokane J R, Ubale A U and Meshram S D, Acta Ciencia, 2006, 4, 477.

6. Shaktawat V, Jain N, Saxsena R, Saxsena N S and Sharma T P, J Optoelectronics Adv Mater., 2007, 9, 2130. 
7. Ferenets M and Harlin A, Thin Solid Films, 2007, 515, 5324-5328.

8. Sangawar V S, Dhokane R J, Ubale A U, Chikhalikar P S and Meshram S D, Bull Mater Sci., 2007, 30, 163.

9. Sangawar V S, Chikhalikar P S, Dhokane R J, Ubale A U and Meshram S D, Bull.Mater Sci., 2006, 29, 413.

10. Shaktawat V, Sharma K and Saxsena N S, J Ovonic Res., 2010, 6, 239.

11. Sangawar V and Adgaonkar C S, Indian J Pure Appl Phys., 1998, 36, 734.

12. Wallace G, Spines G and Leon A, Conductive Electroactive Polymers Intelligent Materials System Second Ed., 2000.

13. Deshmukh S H, Burghate D K, Akhare V P, Deogaokar V S and Deshmukh P T, Bull Mater Sci., 2007, 30(1), 51-56.

14. Zhang Z M and Wan M X, Synth Metals, 2002, 128(1), 83-89.

15. Kumar V, Sachin Kr Sharma Kr, Sharma T P and Singhm V, Optical Materials, 1999, 12, 115-119.

16. Migahed M and Bakr N, J Polym Matter., 1994, 11, 129.

17. Tauc J, Amorphous and Liquid Semiconductors, New York, Plenum, 1974, 159.

18. Raju C L, Rao J L, Reddy B C V and Brahmam K V, Bull Mater Sci., 2007, 30(3), 215-218.

19. Tajeddin B, Rehman R A, Abdullah L C and Ibrahim N A, Yus Aniza Yusof, European J Scientific Res., 2009, 32(2), 223-230. 